\title{
Susceptibility to Peer Influence: Using a Performance-Based Measure to Identify Adolescent Males at Heightened Risk for Deviant Peer Socialization
}

\author{
Mitchell J. Prinstein, \\ Department of Psychology, University of North Carolina at Chapel Hill \\ Whitney A. Brechwald, and \\ Department of Psychology and Neuroscience, Duke University \\ Geoffrey L. Cohen \\ School of Education, Stanford University
}

\begin{abstract}
A substantial amount of research has suggested that adolescents' attitudes and behaviors are influenced by peers; however, little is known regarding adolescents' individual variability, or susceptibility, to peer influence. In this study, a performance-based index from an experimental paradigm was used to directly measure adolescents' susceptibility to peers. A total of 36 adolescent boys participated in a "chat room" experiment in which they ostensibly were exposed to deviant or risky social norms communicated either by high-peer-status (i.e., popular, well-liked) or low-peer-status (i.e., unpopular, disliked) grade mates who actually were electronic confederates. Changes in adolescents' responses before and after exposure to peer norms were used as a measure of peer influence susceptibility. These same adolescents completed a questionnaire assessment at the study outset and again 18 months later to assess their actual engagement in deviant behavior and their perceptions of their best friend's engagement in deviant behavior. Only among adolescents with high levels of susceptibility to high-status peers was a significant longitudinal association revealed between their best friend's baseline deviant behavior and adolescents' own deviant behavior 18 months later. Findings support the predictive validity of a performance-based susceptibility measure and suggest that adolescents' peer influence susceptibility may generalize across peer contexts.
\end{abstract}

\section{Keywords}

peer influence; deviant/delinquent behavior; susceptibility; adolescents

\begin{abstract}
One of the most consistent and potent predictors of adolescents' engagement in aggressive and health-risk behaviors is the extent to which adolescents' peers engage in similar behaviors (Prinstein \& Dodge, 2008). Unfortunately, little is currently known regarding the best way to mitigate such peer influence effects. Given the remarkable difficulties involved in dissuading adolescents from befriending potentially "risky" peers, recent work has focused on an examination of potentially malleable factors that may diminish susceptibility to peer influences. However, little is known regarding individual differences in peer influence susceptibility. While existence of substantial individual variability generally is
\end{abstract}

(C) 2011 American Psychological Association 2011

Correspondence concerning this article should be addressed to Mitchell J. Prinstein, Department of Psychology, University of North Carolina at Chapel Hill, Davie Hall, Campus Box 3270, Chapel Hill, NC 27599-3270. mitch.prinstein@unc.edu. 
acknowledged in adolescents' conformity behavior, the construct of susceptibility to peer influence has eluded thorough examination in the literature.

Susceptibility previously has been examined in several ways. Recently, individual differences in adolescents' responses to potentially influential peer contexts have been studied through examination of moderators (i.e., social-psychological factors, often of the peer-influence target, such as the peer's own level of social status) that alter the magnitude of the longitudinal association between peers' behavior and adolescents' own behavior. However, it is important to note that these social-psychological factors do not measure susceptibility itself or facilitate the study of susceptibility as a discrete developmental construct. In other words, while factors that may affect susceptibility have been examined in some work, researchers still know relatively little about susceptibility per se.

A second approach to the examination of peer influence susceptibility has been to construct and administer questionnaires in which adolescents are asked to report how much they feel they are influenced by peers or, conversely, how competent they feel at resisting peer influences, or how much they endorse attitudes regarding the appropriateness of conformity (e.g., Steinberg \& Monahan, 2007). This approach more directly addresses the construct of peer influence susceptibility itself and has yielded several important preliminary findings (e.g., Steinberg \& Monahan, 2007). Unfortunately, reliance on adolescents' own reports regarding their susceptibility to peer influence may be compromised by adolescents' limited self-awareness of their attitudes and behaviors, particularly among those most likely to be exposed to deviant peer influences (see Hoza, Pelham, Dobbs, Owens, \& Pillow, 2002; Prinstein \& Wang, 2005). Currently, the construct and predictive validity of a self-report measurement approach for peer influence susceptibility are unknown.

A related approach for measuring peer influence susceptibility involves the use of hypothetical scenarios. In studies conducted in the 1970s and 1980s, a commonly used measure of susceptibility was one in which experimenters posed social situations that varied in potential for peer pressure and assessed whether adolescents would endorse behavioral responses that might be viewed as favorable or unfavorable by peers (e.g., Berndt, 1979; Steinberg \& Silverberg, 1986). Important advantages of this approach include the ability to provide some distance from the overt self-evaluation of susceptibility inherent in self-report indices and the ability to manipulate the conditions in which susceptibility could be examined. Early research utilizing a hypothetical scenario approach focused on age and gender differences in susceptibility, with findings suggesting that peer influence susceptibility likely increases at the adolescent transition (Berndt, 1979; Bixenstine, DeCorte, \& Bixenstine, 1976; Steinberg \& Silverberg, 1986).

A final approach used in past research to measure peer influence susceptibility has involved observational or experimental approaches to measure in vivo behavior (i.e., a performancebased approach). Peer influence susceptibility likely is due to implicit processes outside an individual's awareness. Explicit reports of implicit process are subject to bias and misestimation. A performance-based approach, in contrast, partially bypasses this limitation and can offer an important contribution to this literature. Allen, Porter, and McFarland (2006) designed an observational task in which adolescents were asked to make decisions regarding a hypothetical decision-making task first alone and then after being exposed to differing opinions expressed by a peer, in this case, a close friend. Susceptibility was operationalized as the extent to which adolescents changed their initial decision because of their friends' differing opinion. Allen and colleagues (2006) revealed that peer influence susceptibility was associated concurrently with high levels of substance use, sexual activity, and parent-reported externalizing symptoms. Offering preliminary support for the validity of this measure, results also demonstrated that peer influence susceptibility was a significant 
moderator of the concurrent association between substance use by adolescents' friends and by the adolescents themselves.

In the current study, we also used an experimental paradigm to yield an in vivo, performance-based measure of adolescent peer influence susceptibility. Specifically, a simulated Internet "chat room" was constructed in which adolescents believed they were interacting with specific grade mates from their own school but actually were interacting with electronic confederates, or "e-confederates," made to appear to be high- or low-status grade mates. The difference between adolescents' responses before versus during the chat room interaction was used to index peer influence susceptibility. It was anticipated that engagement in deviant behaviors by the adolescents' best friends would be associated with increases in adolescents' own deviant behavior over time, reflecting a peer socialization effect (e.g., Kandel, 1978). Our primary goal in this study was to examine whether this socialization effect would be moderated by adolescents' peer influence susceptibility, assessed with a performance-based measure. Because peer influence was expected to occur only in the high-peer-status condition, we believed that peer influence susceptibility would be a significant moderator only for adolescents whose susceptibility was measured in the high-peer-status e-confederate condition.

\section{Method}

\section{Participants}

A total of 36 White adolescent boys in 11th grade at study outset participated. At an initial time point, a total of 43 White adolescent boys participated in an experimental paradigm we had designed to examine peer influence. These 43 adolescents were drawn from a larger sample of 273 adolescents ( $42 \%$ boys and 58\% girls; $74 \%$ of eligible population) that was demographically representative of the high school from which it was drawn (see Cohen \& Prinstein, 2006).

For the purposes of the experiment, only adolescents with average peer status (as rated on a standard sociometric assessment) were selected to participate. Only White boys were included in the study because we expected that gender and ethnicity would be associated significantly with peer influence susceptibility in this experimental context. Of the 50 White male participants who received standardized peer-nominated social preference and social reputation scores between -1.0 and 1.0 (indicating average likability and popularity among peers, respectively), 43 participants were available for testing within the limited time period available to conduct this deceptive experimental paradigm. No differences were revealed between these 43 selected participants and the seven who were unable to be scheduled. Each of the 43 participants was randomly assigned to a condition in which they participated in a simulated electronic chat room with either high- or low-status e-confederates. Eighteen months later, 36 of these adolescents were available for follow-up testing and were included in current analyses. There were no significant differences between adolescents who participated at one versus both time points on any study variables. Of the 36 participants who completed Time 2 testing, 18 had participated in the high-status condition and 18 had participated in the low-status condition of the experimental paradigm, described in more detail later.

\section{Procedure}

Participation in this study began with adolescents' completion of a sociometric assessment, as well as completion of questionnaires measuring adolescents' engagement in deviant and health-risk behavior. Adolescents also provided pretest responses to the hypothetical scenarios used in the experimental paradigm (each described later). Next, adolescents 
participated in the experimental paradigm that allowed for an in vivo examination of peer influence susceptibility. Last, 18 months later (i.e., at Time 2), adolescents completed a second questionnaire-based assessment.

\section{Measures}

Sociometric assessment-We conducted a standard sociometric assessment using unlimited gradewide peer nominations with all 273 initial participants at Time 1 to measure adolescents' peer acceptance or rejection (i.e., likeability: "Whom do you like most/least" nominations) and peer-perceived popularity (i.e., "Who is most/least popular" nominations) among their peers (see Cohen \& Prinstein, 2006).

\section{Aggressive and health-risk behavior hypothetical scenario instrument-A}

hypothetical scenario instrument was used to assess adolescents' endorsement of aggressive and health-risk behaviors. The measure includes 14 scenarios in which adolescents may have opportunities to engage in physical aggression, verbal teasing, vandalism, or substance use; each item is accompanied by 3- 6 Likert-format behavioral options that reflect increasingly or decreasingly aggressive or risky behavior options. Responses to individual items are standardized (after being reverse coded where appropriate) and then averaged into a composite. Prior results have supported the reliability and validity of this instrument (Cohen \& Prinstein, 2006).

We used results from a gradewide administration of this instrument at Time 1 to determine the normative (i.e., mean) response to each scenario among White male students at the school. "Above average" (i.e., $1 S D$ ) levels of aggressive or risky behavior endorsement later were attributed to either low- or high-status peers in the context of a simulated chat room. These scenarios were presented again during the experimental paradigm (i.e., in the chat room) to determine whether participants may change their responses when in the presence of - and exposed to norms ostensibly communicated by — high- or low-status peers. Changes in adolescents' responses on these items before versus during the chat room interaction were computed and averaged to form a measure of peer influence susceptibility in this study.

Deviant behavior-At both Times 1 and 2, adolescents completed a measure of their engagement in deviant and delinquent behaviors during the past year. This measure includes five items of deviant behavior (i.e., ruined or damaged other people's property or possessions on purpose; stolen something, or tried to steal something, worth less than $\$ 5$; stolen something, or tried to steal something, worth more than $\$ 50$; broken into a car or building to steal something; and been in a physical fight; $a=.76$ ) that have been used in substantial prior research (Centers for Disease Control and Prevention, 2008; Prinstein \& Wang, 2005). A mean score was computed across the Likert scale responses for each item.

Perceptions of best friends' behavior-Using a peer nomination procedure, adolescents were to identify an unlimited number of their closest friends from a roster of their all grade mates as well as a single peer who was their "very best friend." Next, adolescents were asked to report the perceived frequency of their very best friend's engagement in deviant behavior using items identical to those described earlier.

Experimental paradigm-The experimental paradigm simulated an Internet chat room. Participants were told that they would have an opportunity to communicate electronically with three male grade mates who supposedly were working on computers in other school rooms. In reality, the three grade mates in each participant's chat room were preprogrammed, computer-generated e-confederates constructed with the Direct RT 
computer program (Jarvis, 2004). For a thorough description of the experimental paradigm, including elements designed to bolster plausibility, manipulation check, sample items, and debriefing procedures, see Cohen and Prinstein (2006). The description of the paradigm provided here highlights aspects of the procedure critical to the current study.

In the chat room, adolescents were instructed that they would communicate in a specific order (the participant always responded fourth); thus, all participants were first exposed to the responses of the e-confederates before providing their own responses. Adolescents participated in a chat room with either high- or low-status e-confederates. We manipulated the e-confederates' peer status by listing the first name and last initial of three high- or lowstatus peers (as determined from the prior sociometric assessment) as the best friends for each chat room member, as well as three favorable (e.g., listening to music) or unfavorable (e.g., spending time with parents) activities as chat room members' hobbies. A manipulation check confirmed the success of this manipulation (Cohen \& Prinstein, 2006). Everything else between conditions remained completely identical.

After an orientation to the chat room and its members, participants responded to the same set of hypothetical scenarios involving aggression or risky behavior that they had completed during the earlier, large-scale baseline assessment. For each scenario, participants once again selected the behavior that would best characterize their own behavioral response. The items used in the current study were 10 "conformity items" in which e-confederates consistently responded with above average levels of aggressive or risky behavior (i.e., more aggressive or risky than the responses of the majority of White boys in this sample). Participants' responses to each scenario after they had been exposed to the e-confederates' responses were used to measure peer influence.

In a previously published study, results indicated that adolescents in the high-peer-status experimental condition were more likely than adolescents in the low-peer-status condition to endorse high-risk responses to these conformity items, after their pretest responses to these same items were controlled for (Cohen \& Prinstein, 2006). In other words, prior (betweengroup) analyses demonstrated that peer influence toward aggressive or risky behaviors is more likely when such norms are communicated by high-status, as opposed to low-status, peers.

In the current study, a new variable was extracted from this experimental paradigm that has not been computed or analyzed previously. Specifically, a within-subjects difference score was computed for each participant to indicate whether responses to the same hypothetical scenarios presented before versus during the experimental paradigm may change; peer influence susceptibility (i.e., each participant's own deviation in responses) was operationalized as this change. It was predicted that this measure of susceptibility, or variability in adolescents' acquiescence to conformity pressures, would moderate longitudinal peer influence effects.

Calculating susceptibility-Past research has offered at least two options for the computation of discrepancies. A residual score approach involves the average of residualized scores from complementary regression (of pretest scores onto post-chat-room scores, and vice versa; see Chi \& Hinshaw, 2002; De Los Reyes \& Prinstein, 2004). A second approach involves the calculation of a simple difference score for each unstandardized item between pretest and post-chat-room responses (see Rogosa, Brandt, \& Zimowski, 1982). Both computational procedures were used in the current study, yielding discrepancy scores that were nearly identical (i.e., $I \mathrm{~s}>.93$ ), and producing an identical pattern of results in analyses. For ease of interpretation, results with difference scores are presented in the Results section. A mean score was computed across the standardized 
difference scores for all 10 individual items $(a=.62)$. Positive scores on this measure reflected greater susceptibility to peer conformity pressures in the chat room; negative scores reflected resistance to peer influence.

\section{Results \\ Descriptive Analyses}

Results revealed that peer influence susceptibility was a normally distributed variable. Deviant behavior at Time 2 was slightly, positively skewed. Re-analysis with logtransformed variables revealed an identical pattern of results. Higher levels of peer influence susceptibility were associated with higher levels of adolescents' deviant behavior. All correlations and means are presented in Table 1.

\section{Primary Analyses}

We hypothesized a three-way interaction for the longitudinal prediction of adolescents' deviant behavior. A hierarchical multiple regression analysis was conducted with adolescents' Time 2 deviant behavior as a dependent variable. After controlling for adolescents' baseline levels of deviant behavior, we entered three main effects (each centered): the experimental condition to which adolescents' were assigned (i.e., dummy coded; high-peer-status condition $=1$ ), adolescents' perceptions of their best friend's engagement in deviant behavior, and adolescents' peer influence susceptibility (i.e., the difference score between pretest and post-chat-room responses). All possible two-way interactions were entered in a third step, and a three way interaction was entered on a fourth step (see Table 2). As predicted, this three-way interaction was statistically significant. Four slope estimates were computed. For adolescents in the low-peer-status experimental condition, at high levels of peer influence susceptibility, the slope between best friends' baseline deviant behaviors and adolescents' later deviant behavior was -.84 , ns, at low levels of susceptibility, slope was 1.67 , ns. For adolescents in the high-peer-status experimental condition, at high levels of peer influence susceptibility, the slope between best friends' baseline deviant behaviors and adolescents' later deviant behavior was $1.55, p$ $<.05$; at low levels of susceptibility, slope was -1.51 , ns. Note that due to low power, nonsignificant results should be interpreted cautiously.

Because these analyses relied on such a small sample, we conducted several diagnostics to examine the integrity of analyses. Typically, low sample sizes reduce power and increase the risk of Type 2 errors. Thus, it is possible that significance of findings may be underestimated in the results. However, analyses conducted with small sample sizes also may be vulnerable to errors in estimation of effects due to the potential for a single case to overly influence parameter values. This possibility was addressed both by bootstrapping analyses and regression diagnostics. In both cases, no evidence was revealed to suggest that any case was overly influential in estimating parameter values (i.e., all |DFFIT| statistics < 1, all |DFBetas| $<1)$. Last, to ensure that findings were not due to heteroscedasticity in perceptions of best friends' deviance across different levels of adolescents' peer influence susceptibility, we used Levene's statistic. Results confirmed homogeneity of variance in friends' deviance across groups of adolescents who were low, moderate, or high in peer influence susceptibility, (Levene's $=1.97, d f=2, n s$ ).

\section{Discussion}

This study used a performance-based index from an experimental paradigm to measure adolescent males' susceptibility to peer influence. The use of a performance-based index addressed significant limitations in the prior literature on peer influence; the construct of 
susceptibility was measured directly, and data did not rely on adolescents' self-perceptions of their temptation to acquiesce to socialization pressures. In fact, in this study, adolescents' socialization mirrored the type of implicit peer influence process that likely is more common than overt peer pressures (Brown, Bakken, Ameringer, \& Mahon, 2008). Thus, adolescents' susceptibility was measured in a context in which participants probably did not even realize that their attitudes and behaviors were being socialized. This approach for measuring peer influence susceptibility thus benefits from maximal ecological validity.

Results suggested that adolescents who were especially susceptible to changes in their deviant risk attitudes in the context of popular grade mates (i.e., e-confederates) also were especially likely to be susceptible to their best friend's level of deviant behavior over an 18month interval. That is, among adolescents who were more easily socialized in the experimental chat room, the association between their best friend's deviant behavior at Time 1 and their own deviant behavior at Time 2 was statistically significant, after baseline deviance was controlled. However, no longitudinal association was revealed between the best friend's deviance and an adolescent's own deviance among adolescents who evidenced lower susceptibility in the chat room. These results offer three important contributions to the literature.

First, and perhaps most straightforward, the results offer validity data for this performancebased measure of peer influence susceptibility. These longitudinal results, coupled with recent results from a study of concurrent associations (Allen et al., 2006), suggest that this elusive construct indeed can be measured with experimental and observational methods in a way that yields a powerful predictor of adolescents' future behavior.

Second, the results from this study offer an intriguing expansion of researchers' understanding of the construct of susceptibility itself. In the current study, susceptibility was measured in the context of popular grade mates whom adolescents likely did not believe were their own close friends. Yet, this measure proved a significant moderator of best friend socialization effects. Results therefore suggest that adolescents' peer influence susceptibility may be a trait-like construct that transfers across peer contexts or sources of peer influence. This possibility requires much further testing; however, such results may suggest that adolescents who are likely to emulate some peers' behavior will emulate other peers' behavior, including the behavior of those with whom they have close personal relationships. Susceptibility then may be due to a broader desire to be liked by peers, a more global tendency to engage in insufficient self-directed problem-solving skills, or a general lack of other sources of counterinfluence (e.g., parents, teachers) that may dissuade adolescents from engaging in deviant activity rather than a specific characteristic of the peer context that makes distinct relationships more influential than others.

Yet, adolescents do not seem to be susceptible to peers indiscriminately. In the low-peerstatus condition, adolescents were less likely to emulate their peers' attitudes and behaviors and even exhibited some signs of anticonformity (i.e., adopting opposing attitudes; Cohen \& Prinstein, 2006). In this study, results suggested that susceptibility in the low-status condition did not moderate best friend socialization effects longitudinally. Thus, it may be that adolescents' susceptibility is somehow tied to adolescents' desire to emulate desirable peers or favorable role models (Gibbons, Gerrard, \& Lane, 2003).

Third, results offered some suggestion that peer influence susceptibility to deviant attitudes may be associated with adolescents' prior levels of deviance, even within a sample of average-peer-status adolescents without especially high levels of deviant behavior. It is unclear whether deviant behavior may increase susceptibility to peer influence more generally or, perhaps more likely, whether adolescents' susceptibility to conformity 
pressures may be related to prior engagement in thematically similar behaviors. It is reasonable to assume that adolescents who already have engaged in deviant behaviors may be more likely than others to adopt additional deviant attitudes. Conversely, peer influence may be especially unlikely when adolescents are exposed to socialization pressures regarding attitudes that contradict or oppose their own prior behavioral choices. Prevention strategies aimed toward reducing deviant peer influence may be best targeted toward adolescents who already have demonstrated initial proclivities toward deviant behavior that favorable peers encourage; it is these adolescents who may be most susceptible to negative peer influence effects.

It would be beneficial if future studies addressed some of the important limitations of this study. First, replications of this study with larger sample sizes are needed. Although statistical diagnostics did not suggest that the integrity of findings was compromised by the small sample size in this study, studies with larger samples will allow for more accurate parameter estimates. In addition, the study of gender and ethnicity as factors that may moderate the effects of peer influence susceptibility on later peer socialization sorely is needed. Theory suggests that girls may be more influenced within a friendship context, and ethnic minority youths' susceptibility to peers likely will vary as a function of the ethnic distribution within the specific school context (e.g., Rose \& Rudolph, 2006; Steinberg \& Monahan, 2007). Additionally, missing data issues required us to rely on adolescents' perceptions of their best friends' deviant behavior, rather than using a friend-reported measure; this may have inflated associations between adolescents' and their friends' deviant behavior. The use of friends' actual reported behavior as a predictor is necessary in future studies. Last, it should be noted that results in this study revealed only the role of susceptibility to high-status peers, not susceptibility more generally. In future research, it would be interesting to examine whether susceptibility can be broadly defined or whether it is dependent on a specific context of peers (i.e., is susceptibility to high-status peers different from susceptibility to other peers or all peers?).

Overall, this study offers a direct examination of peer influence susceptibility and yielded some new insights into whether adolescents may exhibit individual differences in their conformity to peers when experimentally presented with identical stimuli. These individual differences appeared to be a valuable indicator of susceptibility to peer socialization effects and a strong predictor of adolescents' actual deviant behavior longitudinally.

\section{Acknowledgments}

This research was supported by Grant 1R01 HD055342 from the National Institute of Child Health and Human Development.

\section{References}

Allen JP, Porter MR, McFarland FC. Leaders and followers in adolescent close friendships: Susceptibility to peer influence as a predictor of risky behavior, friendship instability, and depression. Development and Psychopathology. 2006; 18:155-172.10.1017/S0954579406060093 [PubMed: 16478557]

Berndt TJ. Developmental changes in conformity to peers and parents. Developmental Psychology. 1979; 15:608-616.10.1037/0012-1649.15.6.608

Bixenstine VE, DeCorte MS, Bixenstine BA. Conformity to peer-sponsored misconduct at four grade levels. Developmental Psychology. 1976; 12:226-236.10.1037/0012-1649.12.3.226

Brown, BB.; Bakken, JP.; Ameringer, SW.; Mahon, SD. A comprehensive conceptualization of the peer influence process in adolescence. In: Prinstein, MJ.; Dodge, KA., editors. Understanding peer influence processes in adolescence. New York, NY: Guilford; 2008. p. 17-44. 
Centers for Disease Control and Prevention, U. S. Department of Health and Human Services. Youth risk behavior surveillance: United States 2007. Morbidity and Mortality Weekly Report Surveillance Summaries. 2008; 57(SS-4):1-131.

Chi TC, Hinshaw SP. Mother-child relationships of children with ADHD: The role of maternal depressive symptoms and depression-related distortions. Journal of Abnormal Child Psychology. 2002; 30:387-400.10.1023/A:1015770025043 [PubMed: 12109489]

Cohen GL, Prinstein MJ. Peer contagion of aggression and health-risk behavior among adolescent males: An experimental investigation of effects on public conduct and private attitudes. Child Development. 2006; 77:967-983.10.1111/j.1467-8624.2006.00913.x [PubMed: 16942500]

De Los Reyes A, Prinstein MJ. Applying depression-distortion hypotheses to the assessment of peer victimization in adolescents. Journal of Clinical Child and Adolescent Psychology. 2004; 33:325335.10.1207/s15374424jccp3302_14 [PubMed: 15136197]

Gibbons, FX.; Gerrard, M.; Lane, DJ. A social reaction model of adolescent health risk. In: Suls, JM.; Wallston, K., editors. Social psychological foundations of health and illness. Oxford, UK: Blackwell; 2003. p. 107-136.

Hoza B, Pelham WE, Dobbs J, Owens JS, Pillow DR. Do boys with attention-deficit/hyperactivity disorder have positive illusory self-concepts? Journal of Abnormal Psychology. 2002; 111:268278.10.1037/0021-843X.111.2.268 [PubMed: 12004833]

Jarvis, B. DirectRT [Computer software]. New York, NY: Empirisoft Corp; 2004.

Kandel DB. Homophily, selection, and socialization in adolescent friendships. American Journal of Sociology. 1978; 84:427-436.10.1086/226792

Prinstein, MJ.; Dodge, KA. Understanding peer influence in children and adolescents. New York, NY: Guilford; 2008.

Prinstein MJ, Wang SS. False consensus and adolescent peer contagion: Examining discrepancies between perceptions and actual reported levels of friends' deviant and health risk behaviors. Journal of Abnormal Child Psychology. 2005; 33:293-306.10.1007/s10802-005-3566-4 [PubMed: 15957558]

Rogosa D, Brandt D, Zimowski M. A growth curve approach to the measurement of change. Psychological Bulletin. 1982; 92:726-748.10.1037/0033-2909.92.3.726

Rose AJ, Rudolph KD. A review of sex differences in peer relationship processes: Potential trade-offs for the emotional and behavioral development of girls and boys. Psychological Bulletin. 2006; 132:98-131.10.1037/0033-2909.132.1.98 [PubMed: 16435959]

Steinberg L, Monahan KC. Age differences in resistance to peer influence. Developmental Psychology. 2007; 43:1531-1543.10.1037/0012-1649.43.6.1531 [PubMed: 18020830]

Steinberg L, Silverberg SB. The vicissitudes of autonomy in early adolescence. Child Development. 1986; 57:841-851.10.2307/1130361 [PubMed: 3757604] 


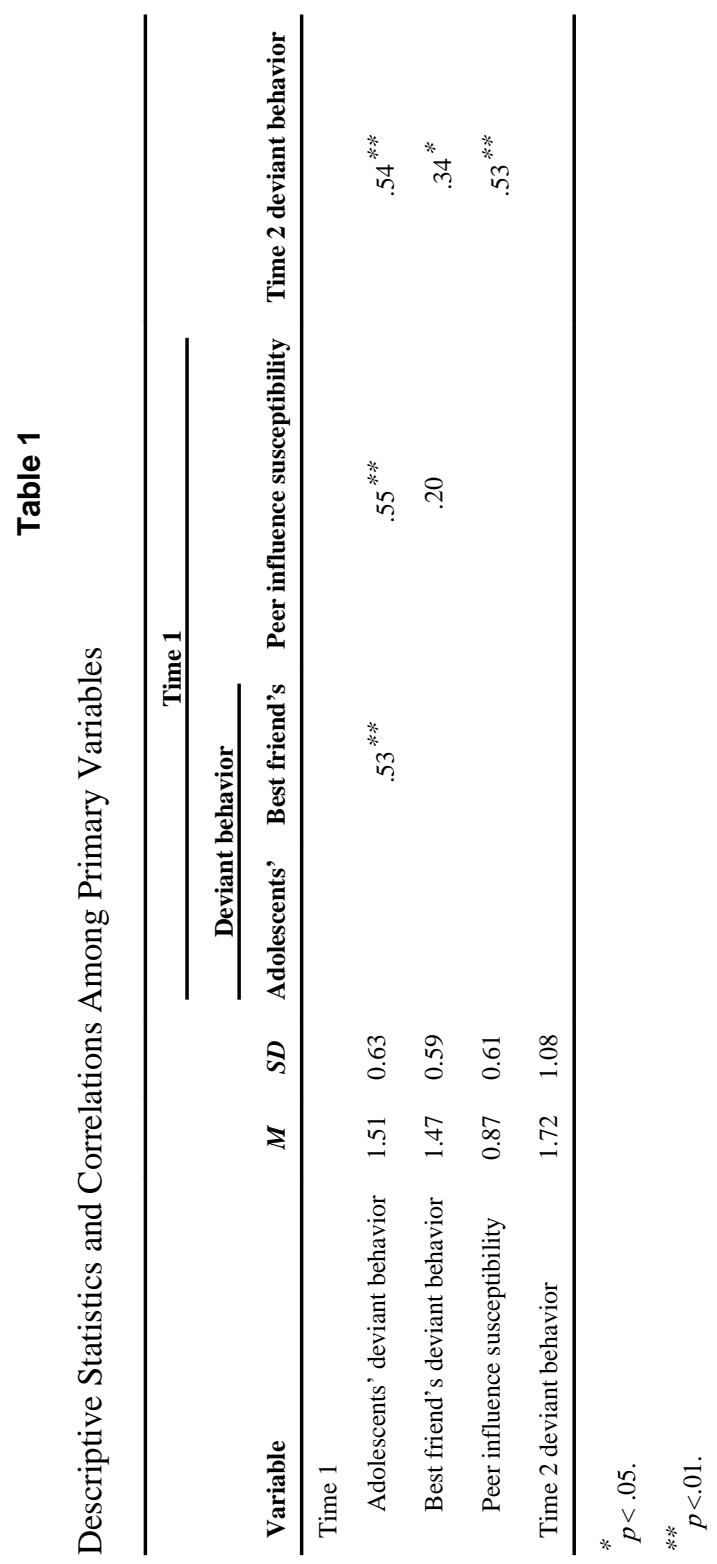




\section{Table 2}

Hierarchical Multiple Regression Results Examining Peer Influence Susceptibility as a Moderator of Best Friend's Deviant Socialization Over 18 Months

\begin{tabular}{|c|c|c|c|c|}
\hline \multirow[b]{2}{*}{ Variable } & \multirow[b]{2}{*}{$\Delta R^{2}$} & \multicolumn{2}{|c|}{ Final $b$} & \multirow[b]{2}{*}{ Final $\beta$} \\
\hline & & $M$ & $S E$ & \\
\hline Step 1 & $.35^{* *}$ & & & \\
\hline Time 1 deviant behavior & & 0.83 & 0.56 & .42 \\
\hline Step 2: Main effects & .06 & & & \\
\hline Best friend Time 1 deviant behavior & & 0.14 & 0.43 & .08 \\
\hline Peer influence susceptibility & & 0.62 & 0.46 & .33 \\
\hline Experimental condition & & 0.18 & 0.37 & 0.8 \\
\hline Step 3: Two-way interactions & .07 & & & \\
\hline Best Friend's Time 1 Deviant Behavior $\times$ Peer Influence Susceptibility & & 1.10 & 0.46 & $.44^{* *}$ \\
\hline Best Friend's Time 1 Deviant Behavior $\times$ Experimental Condition & & 0.22 & 0.68 & .07 \\
\hline Experimental Condition $\times$ Peer Influence Susceptibility & & 0.17 & 0.71 & .06 \\
\hline Step 4: Three-way interaction & $.08^{*}$ & 2.00 & 0.96 & $.51^{*}$ \\
\hline
\end{tabular}

Note. Dependent variable $\times$ Time 2 deviant behavior.

* $p<.05$.

*** $p<.01$ 